Revista Destaques Acadêmicos, Lajeado, v. 9, n. 3, 2017. ISSN 2176-3070

DOI: http://dx.doi.org/10.22410/issn.2176-3070.v9i3a2017.1471

www.univates.br/revistas

\title{
INFLUÊNCIA DO NÍVEL DE ATIVIDADE FÍSICA NA CAPACIDADE FUNCIONAL DE MULHERES IDOSAS
}

\author{
Bruno de Maman ${ }^{1}$, Fabian Arruda Barbosa ${ }^{2}$, Augusto Brugnera ${ }^{3}$, \\ Daniela Beatriz da Silva ${ }^{4}$, Rômulo Augusto Scherer ${ }^{5}$, \\ Jéssica Luana Dornelles da Costa ${ }^{6}$, Carlos Leandro Tiggemann ${ }^{7}$
}

\begin{abstract}
Resumo: O objetivo do presente estudo foi identificar a influência do nível de atividade física na capacidade funcional de mulheres idosas. A amostra foi composta por 15 mulheres com idade entre 60 e 78 anos. Para o nível de atividade física, utilizou-se o Questionário Internacional de Atividade Física (IPAQ, 2005), gerando dois grupos, nos quais as idosas foram caracterizadas como ativas (GATI; $\mathrm{n}=7$ ) e sedentárias (GSED; $\mathrm{n}$ $=8$ ). A avaliação da capacidade funcional consistiu de três testes físicos, teste de subir degraus (DEG), teste de sentar e levantar em uma cadeira (CAD) e o Timed Up and Go (TUG). Houve diferença significativa $(\mathrm{p} \leq 0,05)$ entre os grupos, apenas nas variáveis do nível de atividade física e no teste TUG, sendo em ambas, uma superioridade em favor do grupo ativo. Concluiu-se que o nível de atividade física tem influência no TUG, porém não no DEG e CAD.
\end{abstract}

Palavras-chave: Atividade Física. Capacidade Funcional. Idosos.

\section{INTRODUÇÃO}

O aumento da população idosa no Brasil tornou-se um assunto recorrente e sua discussão acelerou ainda mais a partir da década de 60, quando

1 Graduado em Educação Física Bacharelado - Univates.

2 Graduado em Educação Física Bacharelado - Univates.

3 Acadêmico do Curso de Educação Física Bacharelado - Univates.

4 Acadêmica do Curso de Educação Física Bacharelado - Univates.

5 Graduado em Educação Física Bacharelado - Univates.

6 Graduada em Educação Física Licenciatura - Univates.

7 Doutor em Ciência do Movimento Humano - Universidade Federal do Rio Grande do Sul (UFRGS). 
a queda das taxas de fecundidade alterou a estrutura etária da população (VERAS, 2007; CHAIMOVICZ, 1997). Segundo dados do Instituto Brasileiro de Geografia e Estatística (IBGE), em 2010, os idosos representavam 10\% da totalidade populacional brasileira, sendo que as projeções são que em 2030, este número passe para 19\% (IBGE, 2013).

O envelhecimento leva a mudanças fisiológicas progressivas, perdas substanciais de funções, redução da força muscular devido à perda de massa muscular (sarcopenia) e redução da atividade física, paralelo a estas mudanças, vem o aparecimento de doenças tipicamente crônico-degenerativas que afetam a qualidade de vida desta população (SILVA et al., 2006; PATERSON; WARBURTON, 2010; WILMORE; COSTILL, 1999; VERAS, 2007).

A população de idosos está entre a mais sedentária e inativa da sociedade, o que pode levar a um declínio na qualidade de vida e uma dependência funcional (PATERSON; WARBURTON, 2010). Dados do Diagnóstico Nacional do Esporte mostram que a prevalência do sedentarismo, no Brasil em idosos, é de $64,4 \%$ (BRASIL, 2015).

A atividade física tem sido altamente indicada para prevenção de doenças, declínios físicos, sociais, psicológicos e flexibilidade do idoso (WEINECK, 2003). O exercício físico em idosos, quando aplicado de forma correta, com intensidade, frequência e duração adequada, pode reduzir alguns processos fisiológicos que atuam conjuntamente com o envelhecimento, aumentando a resistência, força muscular e capacidade funcional. Proporcionando maior independência em suas atividades básicas, tornando sua vida mais saudável (DAVINI; NUNES, 2003; GOBBI, 1997).

Entende-se capacidade funcional como a habilidade que as pessoas têm de decidirem e intervirem de forma independente nas suas vidas, no seu dia a dia (MATSUDO, 2000). É um dos importantes marcadores para um envelhecimento bem-sucedido e consequentemente uma melhora na qualidade de vida dos idosos (CIPRIANI et al., 2010). Quando é aplicada uma avaliação da capacidade funcional, geram-se dados que possibilitam identificar o perfil desta população, o que muitas vezes pode auxiliar em determinar planos para retardar ou prevenir as incapacidades (FIEDLER; PERES, 2008).

Uma das formas para retardar as incapacidades provocadas pelo declínio da capacidade funcional é o treinamento de força. Em estudo de Tiggemann et al. (2016), dois grupos de idosas realizaram treinamento de força tradicional e treinamento em velocidade máxima, sendo avaliada a força máxima e as capacidades funcionais. Os autores encontraram melhora significativa na força máxima e nas capacidades funcionais nos dois grupos pesquisados.

O estudo de Cipriani et al. (2010) analisou idosas que já participavam de um programa de atividades físicas. As idosas foram acompanhadas por 10 meses, sendo analisado o Índice de Aptidão Funcional Geral, agilidade/ equilíbrio dinâmico, coordenação, flexibilidade, resistência de força de 
membros superiores e resistência aeróbia geral. O estudo encontrou melhoras na funcionalidade e nas capacidades agilidade/equilíbrio dinâmico e coordenação, sendo que houve manutenção das demais capacidades físicas.

Pode-se constatar que o exercício físico é de suma importância para melhora e manutenção das capacidades físicas de idosos, tendo influência direta na sua qualidade de vida. Os estudos demonstram a efetividade do exercício físico e atividades físicas programadas na melhora da capacidade funcional, e das capacidades físicas de idosos. Porém, poucos estudos têm avaliado a influência da atividade física habitual nestes parâmetros. Sendo assim o objetivo deste estudo é verificar a influência do nível de atividade física na capacidade funcional de mulheres idosas.

\section{MÉTODO}

A pesquisa classificou-se como um estudo transversal quantitativo, no qual foi entregue às participantes um Termo de Consentimento Livre Esclarecido (TCLE), no qual estavam cientes do estudo e concordaram em participar da pesquisa de livre e espontânea vontade. Este estudo foi aprovado pelo Comitê de Ética e Pesquisa (COEP) da UNIVATES, parecer número 1.567.120.

A amostra consistiu de 15 mulheres com idade entre 60 e 78 anos, aparentemente saudáveis, recrutadas via meios de comunicação como: internet, rádio, folders, panfletos, visita a centro de convivência de idosos. Foram adotados como critérios de inclusão para participação do estudo: (1) não ter praticado treinamento de força, no mínimo, por seis meses antes do começo do estudo; (2) ausência de histórico de doenças cardiovasculares (a exceção de hipertensão arterial controlada por medicamento), endócrinas, metabólicas, neuromusculares e articulares (a exceção de quadros leves de artrite e/ou artrose); (3) não estar em tratamento à base de medicamentos de uso contínuo ou eventual que afetasse o equilíbrio. Não houve exclusão de nenhuma participante do estudo.

A amostra foi caracterizada quanto ao nível de atividade física por meio da aplicação do Questionário Internacional de Atividade Física (IPAQ, 2005). O questionário foi aplicado durante entrevista no Laboratório de Fisiologia do Exercício da UNIVATES, sendo preenchido por todas as participantes do estudo, sem interferências ou influências do acadêmico ou do seu orientador. O IPAQ foi desenvolvido para avaliar os níveis da prática de atividade física de diversas populações, que é culturalmente adaptável, sem perder suas especificidades. O questionário mede o tempo gasto em diferentes atividades físicas, com um tempo mínimo de dez minutos e os sujeitos podem ser classificados em três níveis de atividade física: baixo (considerados sedentários), moderado e intenso (considerados ativos), conforme os dados de uma semana habitual (IPAQ, 2005; GUEDES et al., 2005). A partir destes resultados os sujeitos foram estratificados em dois grupos: idosas ativas (GATI; $\mathrm{n}=7$ ) e as sedentárias (GSED; $\mathrm{n}=8$ ). 
Todos os testes foram aplicados por equipe de avaliadores capacitados e aptos, sendo realizados junto ao complexo esportivo da UNIVATES, no turno da tarde. Para minimizar o efeito da aprendizagem, todos os testes foram realizados em dois momentos (teste e retestes) com um intervalo de uma semana entre eles. A avaliação da capacidade funcional consistiu de três testes físicos, baseados em atividades motoras realizadas no dia a dia de idosos, sendo eles: teste de subir degraus (DEG), teste de sentar e levantar em uma cadeira (CAD) e o Timed Up and Go (TUG). Todos os testes funcionais foram realizados por duas tentativas, sempre realizados em velocidade máxima, com intervalo de 3 minutos entre cada tentativa, sendo registrado menor tempo. Um intervalo de vinte minutos foram dados entre cada teste, sendo este protocolo previamente utilizado pela literatura (TIGGEMANN et al., 2016).

No teste DEG, os sujeitos deviam subir um lance de 10 degraus (cada degrau $0,18 \mathrm{~m}$ de altura e $0,26 \mathrm{~m}$ de comprimento) sem o uso do corrimão na velocidade máxima, eles deveriam pisar em um degrau de cada vez. O tempo foi registrado a partir do primeiro contato feito no primeiro degrau, finalizado ao ocorrer o contato com o último degrau. No teste CAD, os sujeitos deveriam levantar e sentar em uma cadeira (assento plano com $43 \mathrm{~cm}$ altura) durante cinco repetições, sendo o tempo registrado ao comando de iniciar o primeiro levantamento (com o sujeito iniciando sentado) e no final (ao sentar após o $5^{\circ}$ levantamento). Os sujeitos deveriam permanecer com os braços cruzados em frente ao tórax, realizar a extensão completa de joelhos, quadris e coluna ao levantar, e ao sentar apoiar completamente as costas no encosto da cadeira. O teste TUG teve início a partir da posição sentada em uma cadeira $(43 \mathrm{~cm}$ de altura, com costas apoiadas), os sujeitos deviam levantar da mesma (sem auxílio das mãos), caminhar e contornar um cone posicionado no solo a $3 \mathrm{~m}$ de distância e voltar a sentar-se (com as costas apoiadas).

Os dados foram apresentados por meio da estatística descritiva (média \pm desvio padrão). A normalidade dos dados foi testada por meio do teste de Shapiro-Wilk e a homogeneidade pelo teste de Levene. A comparação das médias das variáveis entre os grupos foi testada por meio do teste $t$ independente. Um nível de significância de $\mathrm{p} \leq 0,05$ foi utilizado, por meio do pacote estatístico SPSS v. 18.0.

\section{RESULTADOS}

Os resultados estão apresentados na tabela 1, sendo encontradas diferenças significativas $(p \leq 0,05)$ entre os grupos apenas nas variáveis do nível de atividade física e no teste TUG, sendo em ambas, uma superioridade em favor ao grupo ativo. As demais variáveis não apresentaram diferença significativa $(\mathrm{p} \geq 0,05)$. 
Tabela 1 - Estatística descritiva (média e desvio padrão) do grupo sedentárias (GSED; $\mathrm{n}=8$ ) e do grupo ativas (GATI; $\mathrm{n}=7$ )

\begin{tabular}{l|c|c|c}
\hline Variáveis & GSED (n = 8) & GATI $(\mathbf{n}=\mathbf{7})$ & $p$ \\
\hline Idade (anos) & $66,25 \pm 6,52$ & $63,00 \pm 2,89$ & 0,246 \\
\hline Massa Corporal (Kg) & $72,44 \pm 13,33$ & $66,23 \pm 11,34$ & 0,353 \\
\hline Estatura (m) & $1,57 \pm 0,06$ & $1,52 \pm 0,05$ & 0,093 \\
\hline Nível de AF (METS/min/sem) & $353,00 \pm 194,80$ & $1292,43 \pm 306,94$ & $<0,001^{*}$ \\
\hline TUG (seg) & $6,89 \pm 0,88$ & $6,03 \pm 0,48$ & $<0,039^{*}$ \\
\hline CAD (seg) & $12,97 \pm 2,21$ & $11,04 \pm 1,48$ & 0,073 \\
\hline DEG (seg) & $4,31 \pm 0,49$ & $4,03 \pm 0,53$ & 0,307 \\
\hline
\end{tabular}

Legenda: $\mathrm{GSED}=$ grupo sedentárias; $\mathrm{GATI}=$ grupo ativas; $\mathrm{AF}=$ atividade física; $\mathrm{TUG}=$ Timed Up and $\mathrm{Go} ; \mathrm{CAD}$ = sentar e levantar em uma cadeira; $\mathrm{DEG}$ = subir degraus. * diferença significativa entre grupos $(\mathrm{p} \leq 0,05)$.

\section{DISCUSSÃO}

O presente estudo teve como finalidade verificar a influência do nível de atividade física na capacidade funcional de mulheres idosas, sendo evidenciado que as idosas ativas apresentaram melhor desempenho apenas no teste TUG. A realização de atividades físicas é um importante fator para melhora da qualidade de vida de idosos, por retardar ou diminuir as mudanças funcionais e físicas decorrentes do envelhecimento, bem como, contribuir na prevenção de doenças crônicas, melhora nos aspectos psicológicos, mantendoos independentes por mais tempo (GABRIEL; POZZOBON, 2013).

O resultado encontrado no teste TUG corrobora com estudo de Riberio et al. (2013), que comparou o equilíbrio dinâmico de idosos ativos e sedentários, indicando que os idosos ativos realizaram o TUG em tempo significativamente menor que os sedentários. Outro estudo, quando comparou idosos que praticavam atividade física regular e que não praticavam, mostrou que o grupo ativo teve uma média de tempo para o teste de 7,8 segundos e o grupo de idosos sedentários teve média de 13,6 segundos (GUIMARÃES et al., 2004).

O TUG serve para mensurar a mobilidade funcional e equilíbrio dinâmico, mostrando que idosos ativos o realizam em tempo inferior a indivíduos sedentários (PODSIADLO; RICHARDSON, 1991). O estudo de Sousa et al. (2011) indicou que há correlação entre equilíbrio e capacidade funcional em idosas com vestibulopatias periféricas. Esta diferença poderia ser explicada pelo fato da atividade física propiciar ao praticante melhoras na postura e equilíbrio corporal (TEIXEIRA et al., 2008).

No presente estudo, o teste CAD não mostrou diferença significativa entre os grupos. Este resultado é contrário ao estudo de Pereira et al. (2009), que 
comparou a força de membros inferiores e superiores entre idosas fisicamente ativas e sedentárias, indicando valores superiores nos sujeitos ativos. Outros estudos também indicaram que o treinamento funcional ou de força podem melhorar a força de membros inferiores de idosos, repercutindo diretamente no teste CAD (MIRANDA et al., 2016; TIGGEMANN et al., 2016).

No estudo conduzido por Famula et al. (2013), os autores não encontraram diferença significativa entre idosas ativas e sedentárias no teste CAD, e hipotetizaram que o fato de ser ativo em outras idades que antecederam o envelhecimento, pode ter alguma influência na força, capacidade funcional e equilíbrio de idosos. Além deste aspecto que poderia explicar o presente resultado, poderia-se especular que outro fator que pode ter influenciado no resultado seria o fato de todas as idosas serem aparentemente saudáveis, garantido boa execução do teste de ambos os grupos.

Ainda, o presente estudo não foi encontrou diferenças significativas em idosas ativas e sedentárias no teste DEG. Este resultado corrobora com um estudo que comparou o efeito de um programa de 10 semanas de atividades físicas em idosos sobre o desempenho em atividades da vida diária, onde os dados pré e pós treinamento não apresentou diferença significativa no teste DEG (HERNANDES; BARROS, 2004).

Apenas a realização de atividades físicas diárias pode não ser o suficiente para a melhora da capacidade funcional de idosos, e neste sentido, outros estudos têm sugerido que somente quando há um treinamento específico é que existe melhora nestes parâmetros (HERNANDES; BARROS, 2004). Tiggemann et al. (2016) realizaram uma intervenção de 12 semanas de treinamento de força com idosas, encontrando melhoras significativas após o treinamento no teste de subir degrau, indicando que a força muscular é determinante no desempenho deste teste.

\section{CONCLUSÃO}

Desta forma, podemos concluir que o nível de atividade física tem influência no TUG, porém não no DEG e CAD. Possivelmente o TUG esteja mais associado ao equilíbrio dinâmico, e desta forma, indivíduos ativos podem vir a obter um melhor resultado neste teste. Em contrapartida, os testes de CAD e DEG não foram influenciados pela atividade física habitual das idosas, sendo que a força muscular de membros inferiores ter um papel mais decisivo, e para tanto, treinamentos específicos seriam necessários.

\section{REFERÊNCIAS}

BRASIL. Ministério do Esporte. Diesporte - Diagnóstico Nacional do Esporte. Brasília, 2015. 
CHAIMOWICZ, Flávio. A saúde dos idosos brasileiros às vésperas do século XXI: problemas, projeções e alternativas. Revista de Saúde Pública, v.31, n.2, p.184-200, 1997.

CIPRIANI, Natália C. S. et al. Aptidão funcional de idosas praticantes de atividades físicas. Revista Brasileira de Cineantropometria e Desenvolvimento Humano, v.12, n.2, p.106-111, 2010.

DAVINI, Rafael; NUNES, Carolina V. Alterações no sistema neuromuscular decorrentes do envelhecimento e o papel do exercício físico na manutenção da força muscular em indivíduos idosos. Revista Brasileira de Fisioterapia, v.7, n.3, p.201-207, 2003.

FAMULA, Anna et al. Previous physical activity and body balance in elderly people. Biology of Sport, v. 30, n. 4, p. 311-315, 2013.

FIEDLER, Mariarosa M.; PERES, Karen G. Capacidade funcional e fatores associados em idosos do Sul do Brasil: um estudo de base populacional. Caderno de Saúde Pública, v. 24, n. 2, p. 409-415, 2008.

GABRIEL, Karin; POZZOBON, Adriane. Efeito da atividade física na depressão e na qualidade de vida de idosos. Cinergis, v.14, n.2, 2013.

GUIMARÃES, Laiz H. C. T. et al. Comparação da propensão de quedas entre idosos que praticam atividade física e idosos sedentários. Revista Neurociências, v. 12, n. 2, 2004.

GOBBI, Sebastião. Atividade física para pessoas idosas e recomendações da Organização Mundial de Saúde de 1996. Revista Brasileira de Atividade Física e Saúde, v. 2, n. 2, p.41-49, 1997.

GUEDES, Dartagnan P. et al. Reprodutibilidade e validade do Questionário Internacional de Atividade Física em adolescentes. Revista Brasileira de Medicina do Esporte, v. 11, n. 2, p.151-158, 2005.

HERNANDES, Elizabeth S. C.; BARROS, Jônatas F. Efeitos de um programa de atividades físicas e educacionais para idosos sobre o desempenho em testes de vida diária. Revista Brasileira de Ciência e Movimento, v. 12, n. 2, p.43-50, 2004.

INSTITUTO BRASILEIRO DE GEOGRAFIA E ESTÁTICA - IBGE. Disponível em: <http:/ / www.ibge.gov.br/home/estatistica/populacao/projecao_da_ populacao/2013/default_tab.shtm>. Acesso em: 26 de set. 2016.

IPAQ (2005). Guidelines for Data Processing and Analysis of the Internacional Physical Activity Questionnaire. Disponível em: <http:/www.ipaq.ki.se/>. Acesso em: 26 de set. 2016.

MATSUDO, Sandra M. Avaliação do idoso: física e funcional. Londrina: Midiograf; 2000 . 
MIRANDA, Laíse V. et al. Efeitos de 9 semanas de treinamento funcional sobre índices de aptidão muscular de idosas. Revista Brasileira de Prescrição e Fisiologia do Exercício, v. 10, n. 59, p. 386-394, 2016.

PATERSON, Donald H.; WARBURTON, Darren E. R. Physical activity and functional limitations in older adults: a systematic review related to Canada's Physical Activity Guidelines. International Journal of Behavioral Nutrition and Physycal Activity, v. 7, n. 38, 2010.

PEREIRA, Fábio D. et al. Comparação da força funcional de membros inferiores e superiores entre idosas fisicamente ativas e sedentárias. Revista Brasileira de Geriatria e Gerontologia, v. 12, n. 3, p.417-427, 2009.

PODSIADLO, Diane; RICHARDSON, Sandra. The Timed "Up \& GO": a test of basic functional mobility for frail elderly persons. Journal of the American Geriatrics Society, v. 39, n. 2, p.142-148, 1991.

RIBEIRO, Fernando et al. Impacto da prática regular de exercício físico no equilíbrio, mobilidade funcional e risco de queda em idosos institucionalizados. Revista Portuguesa de Ciências do Desporto, v.9, n.1, p.36-42, 2009.

RIBEIRO, Renata F. et al. Avaliação do equilíbrio dinâmico em idosos ativos e sedentários. Fisioterapia Brasil, v.14, n. 5, 2013.

SILVA, Tatiana A. A. et al. Sarcopenia Associada ao Envelhecimento: Aspectos Etiológicos e Opções Terapêuticas. Revista Brasileira de Reumatologia, v .46, n. 6, p.391-397, 2006.

SOUSA, Raquel. F. et al. Correlation between the body balance and functional capacity from elderly with chronic vestibular disorders. Brazilian Journal of Otorhinolaryngology, v. 77, n. 6, p.791-798, 2011.

TEIXEIRA, Clarissa S. et al. Equilíbrio corporal e exercícios físicos: uma investigação com mulheres idosas praticantes de diferentes modalidades. Acta Fisiátrica, v.15, n.3, p.154-157, 2008.

TIGGEMANN, Carlos L. et al. Effect of traditional resistance and power training using rated perceived exertion for enhancement of muscle strength, power, and functional performance. The Official Journal of the American Aging Association, v. 38, n. 42, p.1-12, 2016.

VERAS, Renato. Fórum Envelhecimento populacional e as informações de saúde do PNAD: demandas e desafios contemporâneos. Introdução. Caderno de Saúde Pública, v. 23, n. 10, p. 2463-2466, 2007.

WEINECK, Jürgen. Atividade física e esporte: para quê? Barueri, SP: Manole, 2003.

WILMORE, Jack H.; COSTILL, David L. Fisiologia do esporte e do exercício. 1. ed., São Paulo: Manole Ltda, 2001. 